\title{
Multifunctional two-dimensional glassy graphene devices for vis-NIR photodetection and volatile organic compound sensing
}

\author{
Xiao $\mathrm{Li}^{1,2 \dagger}$, Xiao $\mathrm{Dai}^{3 \dagger}$, Hao Xu ${ }^{1,2^{*}}$, Kai Shen ${ }^{1}$, Jian Guo ${ }^{4}$, Caihong $\mathrm{Li}^{2}$, Guifu Zou ${ }^{3^{*}}$, \\ Kwang-Leong Choy ${ }^{5^{*}}$, Ivan P. Parkin ${ }^{4}$, Zhengxiao Guo ${ }^{4,6,7}$, Huiyun Liu ${ }^{1}$ and Jiang Wu ${ }^{1,2}$
}

\begin{abstract}
Multifunctional devices are of great interest for integration and miniaturization on the same platform, but simple addition of functionalities would lead to excessively large devices. Here, the photodetection and chemical sensing device is developed based on two-dimensional (2D) glassygraphene that meets similar property requirements for the two functionalities. An appropriate bandgap arising from the distorted lattice structure enables glassy graphene to exhibit comparable or even improved photodetection and chemical sensing capability, compared with pristine graphene. Due to strong interactions between glassy graphene and the ambient atmosphere, the devices are less sensitive to photoinduced desorption than the ones based on graphene. Consequently, the few-layer glassy graphene device delivers positive photoresponse, with a responsivity of $0.22 \mathrm{~A} \mathrm{~W}^{-1}$ and specific detectivity reaching $\sim 10^{10}$ Jones under $405 \mathrm{~nm}$ illumination. Moreover, the intrinsic defects and strain in glassy graphene can enhance the adsorption of analytes, leading to high chemical sensing performance. Specifically, the extracted signalto-noise-ratio of the glassy graphene device for detecting acetone is 48 , representing more than $50 \%$ improvement over the device based on graphene. Additionally, bias-voltage- and thickness-dependent volatile organic compound (VOC) sensing features are identified, indicating the few-layer glassy graphene is more sensitive. This study successfully demon-
\end{abstract}

strates the potential of glassy graphene for integrated photodetection and chemical sensing, providing a promising solution for multifunctional applications further beyond.

Keywords: glassy graphene, multifunctional device, photodetection, volatile organic compounds, liquid sensing

\section{INTRODUCTION}

An ever-increasing demand for system integration and miniaturization drives the development of multifunctional devices that can efficiently respond to different physical and chemical signals [1,2]. Particularly of interest are high-performance photodetectors and chemical sensors. The former are widely applied in optical communication, imaging, spectroscopy, biosensing, night vision and so on [3-6]. The latter are increasingly in demand in many fields such as healthcare, environmental monitoring and risk warning [7-9]. Both are critical for personalized medical-care, public safety, security and the Internet of Things (IoT). Such applications pose more stringent requirements for the functionalities of materials, especially their optical and chemical properties. It is noted that the requirements for high-performance photodetection and chemical sensing are similar, including an appropriate bandgap. For example, metal oxide semi-

\footnotetext{
${ }^{1}$ Department of Electronic and Electrical Engineering, University College London, Torrington Place, London WC1E 7JE, United Kingdom

${ }^{2}$ Institute of Fundamental and Frontier Sciences, University of Electronic Science and Technology of China, Chengdu 610054, China

${ }^{3}$ School of Energy, Soochow Institute for Energy and Materials Innovations, and Key Laboratory of Advanced Carbon Materials and Wearable Energy Technologies of Jiangsu Province, Soochow University, Suzhou 215006, China

${ }^{4}$ Department of Chemistry, University College London, 20 Gordon Street, London WC1H 0AJ, United Kingdom

${ }^{5}$ Institute for Materials Discovery, University College London, Roberts Building, Malet Place, London WC1E 7JE, United Kingdom

${ }^{6}$ Departments of Chemistry and Mechanical Engineering, The University of Hong Kong, Hong Kong SAR, China

${ }^{7}$ Zhejiang Institute of Research and Innovation, The University of Hong Kong, Qingshan Lake SciTech City, Hangzhou 311305, China

These authors contributed equally to this paper.

* Corresponding authors (emails: hao.xu.15@uestc.edu.cn (Xu H); zouguifu@suda.edu.cn (Zou G); k.choy@ucl.ac.uk (Choy KL))
} 
conductors, especially $\mathrm{ZnO}$ nanostructures, have been employed for high-performance UV photodetection and gas sensing in a single device, due to attractive combination of electrical and optical properties such as direct bandgap $(\sim 3.37 \mathrm{eV})$, high exciton binding energy $(\sim 60 \mathrm{meV})$, and high electron mobility [2,10-13]. However, multifunctional devices with both visible-to-near infrared (vis-NIR) photodetection and volatile organic compound (VOC) liquid sensing have yet to be achieved thus far. Particularly, it has been demonstrated that liquid VOC detection can be applied as a vital base for simple, rapid and effective disease diagnosis [14,15], and the identification of acetone in human blood, urine and sweat samples has been regarded as a biomarker for diabetes mellitus [14-18].

Besides metal oxide semiconductors, two-dimensional (2D) materials hold great promise for novel multifunctional devices owing to their outstanding electronic, optoelectronic, mechanical and thermal properties [12,19-21]. The photodetectors based on $2 \mathrm{D}$ materials and their heterostructures have been widely reported previously, exhibiting good performance [4,22-24]. Specially, ultrahigh carrier mobility of graphene is particularly attractive for high-speed photodetection [25-27]. However, these photodetectors suffer from limited responsivity due to low light absorption as well as the gapless electronic structure [28-30]. Attributed to its good electrical conductivity and mechanical strength, graphene has been used for chemical sensing with single molecule resolution and low intrinsic noise [31]. Nevertheless, the gapless electronic structure, lack of dangling bonds and challenges in large-scale synthesis hinder pristine graphene from industrial considerations in the field. On the other hand, an appropriate bandgap, good electrical conductivity, a large surface-to-volume ratio and strong affinity to analytes are all ideal properties for photodetection and chemical sensing.

Alternatively, as an emerging graphene derivative, 2D glassy graphene with a distorted lattice is dominated by the Stone-Wales (SW) defects with twisted C-C bonds by $90^{\circ}$ [32]. The detailed synthesis method, distorted lattice structure, basic electrical and optical properties of glassy graphene can be found in our previous reports $[32,33]$. Originating from its unique atomic structure, an intrinsic bandgap is introduced, and the width of the gap increases with the value of the defect ratio [33]. Especially, in a glassy graphene model of $8 \times 8$ supercell with one SW defect, a bandgap of $30 \mathrm{meV}$ emerges at the Dirac point [33]. The intrinsic energy bandgap can be expected to boost responsivity and chemical sensing. It is well known that opening the bandgap is one of the solutions to improve responsivity of photodetectors based on graphene. Also, introducing defects or functional groups have been utilized to enhance the chemical adsorption of graphene $[26,34,35]$. The glassy graphene could shun the pressure for complex growth and fabrication processes to open an energy bandgap in graphene and related devices. Besides, as reported, glassy graphene shares excellent electrical conductivity, transparency, stability and flexibility with graphene $[33,36]$, and thus it is expected to be a desirable candidate for multifunctional demonstrations. More importantly, the facile and large-scale synthesis of glassy graphene is essential for assembling device arrays and low-cost mass production. Hence, glassy graphene satisfies the above requirements for performance enhancement of both photodetection and chemical sensing in a single structural platform.

Here, multifunctional glassy graphene devices with high photodetection and VOC sensing performance are reported. The glassy graphene with an intrinsic bandgap results in strong light absorption, yielding high photocurrent in the vis-NIR range. Importantly, the devices are less affected by the photoinduced desorption, demonstrating a positive photoresponse (PPR), compared with the negative photoresponse (NPR) observed in the pristine graphene device of the same configuration. The responsivity and specific detectivity are obtained to be $0.22 \mathrm{~A} \mathrm{~W}^{-1}$ and $\sim 10^{10}$ Jones under $405 \mathrm{~nm}$ illumination for the glassy graphene device. In terms of liquid VOC sensing, glassy graphene devices show real-time responses to various volatile liquids. Attributed to the defects, glassy graphene devices exhibit comparable or even superior VOC detection capability, compared with graphene devices. For instance, a glassy graphene device shows a signal-to-noise-ratio (SNR) of $48, \sim 50 \%$ improvement superior to the graphene device.

\section{EXPERIMENTAL SECTION}

\section{Material synthesis and characterizations}

Inch-size glassy graphene thin films were grown on quartz by a simple and cost-effective polymer-assisted deposition (PAD) method, and the detailed synthesis process was presented in our reported work [32]. By tuning of the glucose concentration in the precursor, glassy graphene films with different thicknesses were obtained. The monolayer graphene as a reference material was purchased from XFNANO.

Atomic force microscopy (AFM) and current sensing AFM (CS-AFM) were carried out on the 2-nm- and 
25-nm-thick glassy graphene films using a Keysight 5600LS with the contact mode in air, and those with other thicknesses were characterized by a Bruker Dimension Icon with the tapping mode in air. The crystalline structure was examined under high-resolution transmission electron microscopy (HR-TEM) with an FEI Tecnai F20 system. Optical microscopy images were obtained using a Carl Zeiss Axioskop Microscope. X-ray photoelectron spectroscopy (XPS) measurement was performed by a Thermo Scientific Ka photoelectron spectrometer using monochromatic Al Ka radiation. Higher-resolution scans were recorded for the principal peaks of $\mathrm{Au} 4 \mathrm{f}$, Si 2p, Ni 2p, O 1s and C 1s at a pass energy of $50 \mathrm{eV}$. UV photoelectron spectroscopy (UPS) was recorded on a thermo theta probe spectrometer with a base pressure of 5 $\times 10^{-10}$ mbar using He (I) UV light with photon energy of $21.2 \mathrm{eV}$. Photoelectron kinetic energy was measured using a hemispherical analyzer with angular acceptance over a solid angle of $60^{\circ}$. Samples were biased at $-9 \mathrm{~V}$ relative to the spectrometer electron optics. The sessile drop experiments were carried out to determine the contact angles using Attension Theta Lite at room temperature in ambient conditions. A charge-coupled device (CCD) camera was used to take images of acetone droplets (50 images per second), followed by an automatic calculation of the static contact angles involving the OneAttension Version 2.3 software. Raman spectroscopy measurement was performed on a Renishaw microRaman system using a 532-nm excitation laser and $1800 \mathrm{~g} \mathrm{~mm}^{-2}$ grating.

\section{Device fabrications}

The glassy graphene arrays with different channel thicknesses were fabricated by the standard photolithography process. $\mathrm{Ti} / \mathrm{Au}(10 / 100 \mathrm{~nm})$ were deposited as electrodes by electron-beam evaporation. To achieve optimum performance, the control graphene devices were fabricated using $\mathrm{Ni}$ rather than $\mathrm{Ti}$ as the contact material for smaller contact resistance $[37,38] . \mathrm{Ni} / \mathrm{Au}(10 / 100 \mathrm{~nm})$ contact pads were deposited by thermal evaporation for graphene devices.

\section{Device characterizations}

\section{Photodetection measurements}

The photoresponse measurements were conducted using Keithley 4200 in two-terminal configuration with the channel length $\times$ width of $100 \times 100 \mu \mathrm{m}^{2}$. Laser diodes with wavelengths of 450,520, 635, 780, 808 and $980 \mathrm{~nm}$ were employed as the external illumination sources, with the corresponding power intensities of $4.9,0.4,4.1,4.5$, 9.9 and $4.3 \mathrm{~mW} \mathrm{~cm}^{-2}$, respectively. The power density of the $405-\mathrm{nm}$ laser diode was tunable from 1.23 to $27.4 \mathrm{~mW} \mathrm{~cm}^{-2}$.

\section{Chemical sensing measurements}

For the VOC sensing measurements, the channel between two contact pads of $100 \times 100 \mu \mathrm{m}^{2}$ was used as the sensing channel. Acetone, isopropyl alcohol (IPA) or methanol of $30 \mu \mathrm{L}$ volume each was dropped individually by a micropipette onto the devices, and channel current values were measured continuously by the Keithley 4200 at ambient conditions. The devices were cleaned by deionized (DI) water and blown by $\mathrm{N}_{2}$ after each measurement cycle to make sure the initial channel current $I_{0}$ returning to the original level. The SNR values for sensing acetone, IPA and methanol were calculated by the Matlab

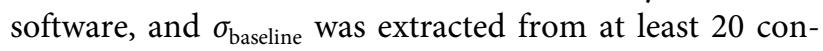
tinuous points. All measurements were conducted at room temperature $\left(25^{\circ} \mathrm{C}\right)$. The acetone was bought from Honeywell with a density of $0.79 \mathrm{~g} \mathrm{~cm}^{-3}$. The IPA and methanol were purchased from $\mathrm{BDH}$, with densities of 0.785 and $0.792 \mathrm{~g} \mathrm{~cm}^{-3}$, respectively.

\section{RESULTS AND DISCUSSION}

As noted in Fig. 1a-c, AFM surface topographies show good uniformity and smoothness of glassy graphene (roughness $<1.04 \mathrm{~nm}$ ). The thicknesses of the glassy graphene films (see insets) extracted from the corresponding AFM height profiles are 0.9, 1.5 and $2 \mathrm{~nm}$, respectively, indicating film synthesis with sub-nanometer precision. The AFM images of multilayer glassy graphene are displayed in Fig. S1a, b, about 5.4 and $25 \mathrm{~nm}$ thick, respectively. Additionally, CS-AFM was performed on the 2 - and 25-nm glassy graphene, respectively. The spatial current maps in Fig. 1d and Fig. S1c, show the compact, uniform and electrically conductive surfaces of glassy graphene (the pink areas) for both thin and thick films. The crystal structure of the synthesized glassy graphene was examined by HR-TEM as shown in Fig. 1e, which clearly reveals the distorted lattice of the few-layer glassy graphene. Evidently, such structural difference at the atomic level between glassy graphene and pristine graphene can lead to dissimilar properties such as the presence of bandgap and lattice strain for the glassy graphene, which are beneficial to photodetection and VOC sensing $[39,40]$. Furthermore, digital camera images of glassy graphene device arrays are given in Fig. If and Fig. S2, respectively. Good transparency was observed for few-layer glassy graphene $(0.9,1.5$ and $2 \mathrm{~nm})$ devices 
$\mathbf{a}$

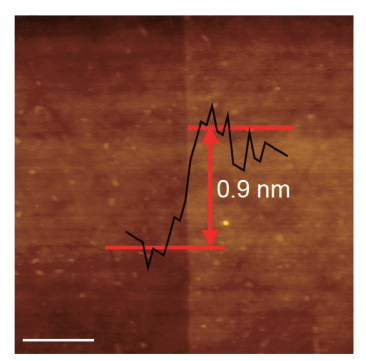

d

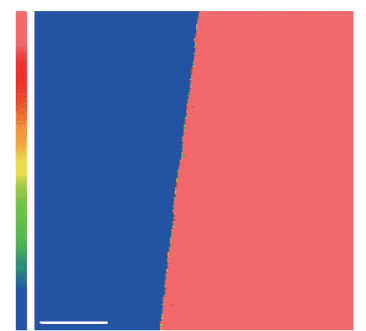

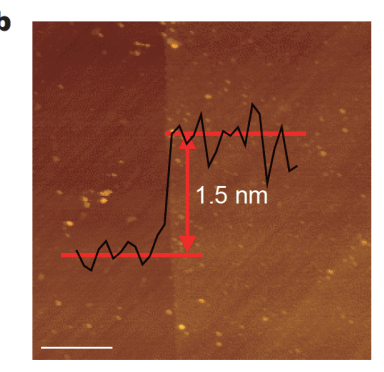
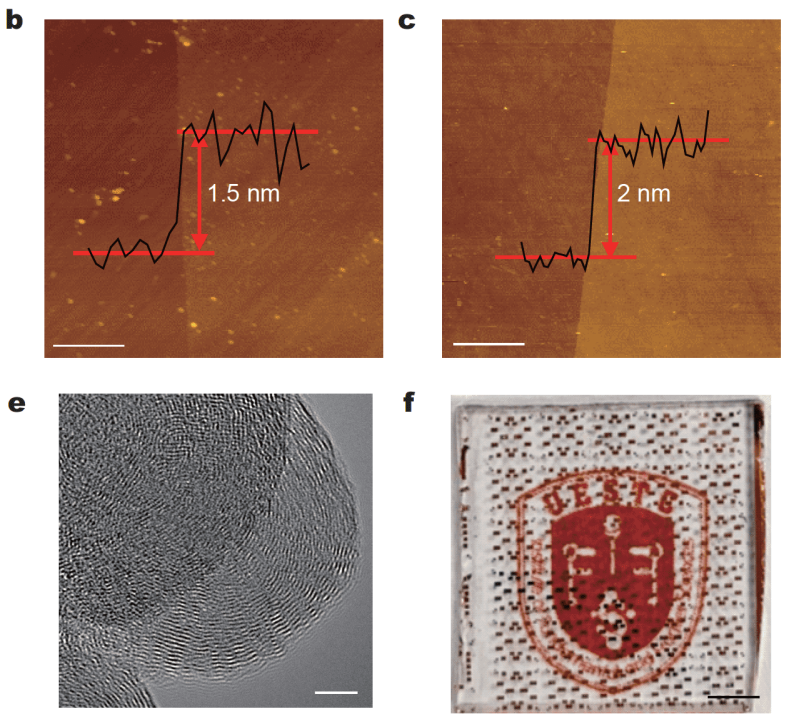

Figure 1 Material characterization results for glassy-graphene and device array images. AFM images of surface morphology for (a) 0.9-nm, (b) 1.5-nm and (c) 2-nm glassy-graphene thin films, obtained at the channel edges. The scale bar is $1 \mu \mathrm{m}$. The insets are the height profiles corresponding to the film thicknesses. (d) CS-AFM image of 2-nm glassy-graphene, scanned at the same area as (c) and determined from the spatial current mapping. The scale bar is $1 \mu \mathrm{m}$, and the color bar denotes the increased current from blue to pink. (e) HR-TEM image of the few-layer glassygraphene. The scale bar is $5 \mathrm{~nm}$. (f) Digital photograph of 0.9-nm glassy-graphene device arrays on quartz, showing good transparency. The scale bar is $2 \mathrm{~mm}$.

(FGD), while the channels were clearly visible to the naked eye for multilayer glassy graphene $(25 \mathrm{~nm})$ devices (MGD).

\section{Vis-NIR photodetection}

The photoresponse behavior of the glassy graphene was studied under visible light illumination using a lateral photoconductor device configuration as shown in Fig. 2a. The transmittance spectra of the glassy graphene films with various thicknesses are shown in our previous work [32,33], where the 0.9 -nm-thick glassy graphene shows transmittance of $\sim 89 \%$, and the 1.2-nm-thick glassy graphene thin film exhibits transmittance of $\sim 65 \%-70 \%$ in the range of $350-850 \mathrm{~nm}$. Initially, the photoresponsive characteristics of the $1.5-\mathrm{nm}$ glassy graphene photodetectors were identified under $405-\mathrm{nm}$ illumination at room temperature. The linear relationship in Fig. $2 b$ indicates the ohmic contact between $\mathrm{Ti} / \mathrm{Au}$ and the glassy graphene channel, and a good conductivity of $270 \mathrm{~S} \mathrm{~m}^{-1}$ was identified. Fig. $2 \mathrm{c}$ shows the time-resolved photoresponse at $1 \mathrm{~V}$ bias voltage, illustrating a reproducible photo-switching behavior. The device responded to illumination with an increasing photocurrent $\left(I_{\mathrm{ph}}=42 \mathrm{nA}\right)$, showing a PPR. Corresponding responsivity $(R$, defined as $R=I_{\mathrm{ph}} / P$, where $P$ denotes the incident optical power [41]) of $0.22 \mathrm{~A} \mathrm{~W}^{-1}$ was achieved, comparable to conventional monolayer graphene photodetectors without further treatment $[25,42,43]$. The good responsivity per- formance could be attributed to the prolonged carrier lifetime, which results from the structural defects of the glassy graphene. Specific detectivity is defined as $D^{*}=$ $R\left(S / 2 e I_{\text {dark }}\right)^{0.5}$ by assuming shot noise from the dark current $\left(I_{\text {dark }}\right)$ as the major contributor to the total noise, where $S$ is the effective area of the device and $e$ is the elementary charge $[33,44]$. $D^{*}$ of $\sim 10^{10}$ Jones can be estimated for $405 \mathrm{~nm}$ excitation. Considering the $1 / f$ noise, more accurate $D^{*}$ should be obtained. The slow response speed could be attributed to the defects within glassy graphene and the adsorbed molecules from air acting as scattering centers, which are commonly observed in photodetectors based on graphene and other 2D materials $[33,45]$. The photogenerated electron-hole pairs were produced, separated and collected by the electrodes under light illumination to increase the current; however, a slightly decreased peak-current trend (pink dotted line) was observed under a few cycles of illumination as shown in Fig. 2c. It was caused by relatively strong photoinduced desorption on the glassy graphene surface. Similar to the reported p-doping effect of graphene after exposure to air $[46,47]$, species such as water and oxygen molecules were adsorbed on the surface of few-layer glassy graphene, resulting in downward shift of the Fermi level towards the valence band maximum (VBM). Upon laser irradiation, some small molecules were desorbed from the surface, leading to upward shift of the Fermi level towards the conduction band minimum (CBM) and reducing the hole 
a
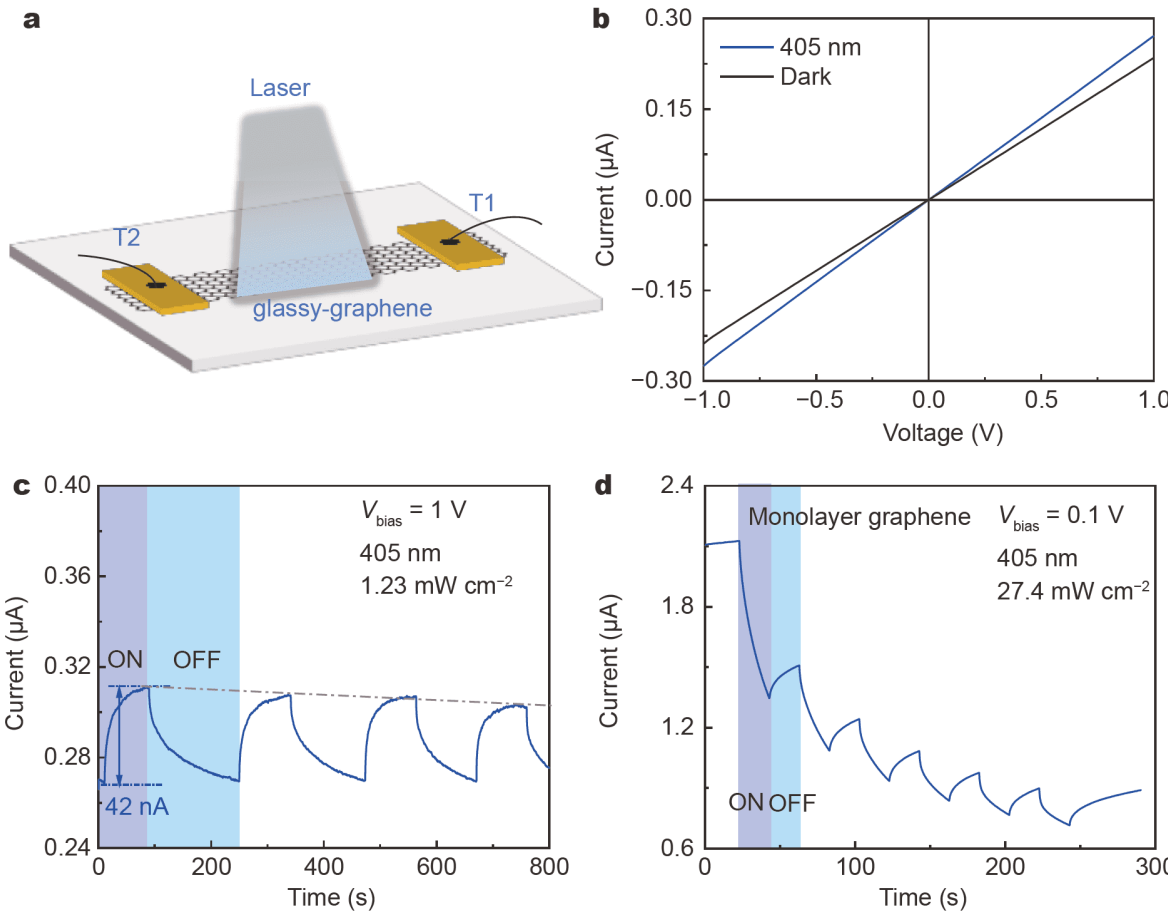

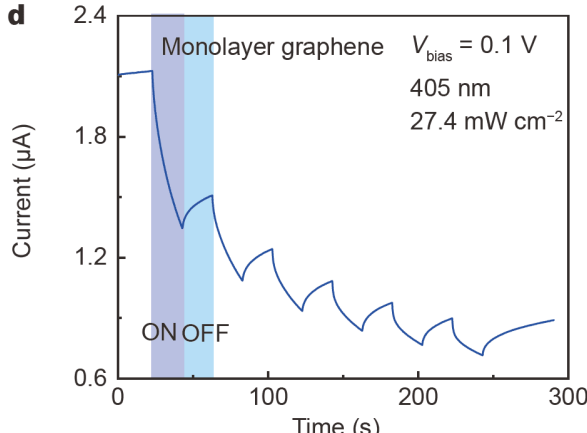

Figure 2 Photoresponsive performance results for few-layer glassy-graphene photodetectors, in comparison to monolayer graphene photodetectors. (a) Schematic configuration of the glassy-graphene photodetector under illumination. (b) $I-V$ characteristic curves of the glassy-graphene photodetector in dark and under $405 \mathrm{~nm}$ illumination, with a power density of $1.23 \mathrm{~mW} \mathrm{~cm}^{-2}$. (c) Photoresponse dynamics with laser on/off switching for glassy-graphene photodetector $\left(1.5 \mathrm{~nm}\right.$ ) as illuminated by $405 \mathrm{~nm}$ laser at $1.23 \mathrm{~mW} \mathrm{~cm}{ }^{-2}$. The applied constant voltage is $1 \mathrm{~V}$. (d) Photoresponse dynamics of the monolayer graphene photodetector as illuminated by $405 \mathrm{~nm}, 27.4 \mathrm{~mW} \mathrm{~cm}^{-2}$. The applied constant voltage is $1 \mathrm{~V}$.

concentration, thus decreasing the current of the glassy graphene device [48]. As a result, current under illumination increased as the photogeneration of electron-hole pairs dominated the process, along with the current peak dropping with time.

For comparison, the performance of a pristine graphene device of the same configuration was characterized and presented in Fig. 2d. The Raman spectrum of monolayer graphene is displayed in Fig. S3, according to which the low defect density can be deduced. In contrast to PPR in glassy graphene, an NPR was observed under $405 \mathrm{~nm}$ illumination at room temperature. The current decreased under illumination as the photoinduced desorption of small molecules dominated the process, and reabsorption of small molecules was responsible for the increased current at dark. The opposite photoresponse performance of glassy graphene and graphene devices was associated with the competition between the photogeneration of electron-hole pairs and the photoinduced desorption of small molecules [49]. According to previous studies, SW defects enhanced the adsorption of the analyte and exhibited larger binding energy and smaller bond distance $[48,50]$. Therefore, SW defects in glassy graphene acted as more stable binding sites with respect to the perfect sites in pristine graphene for small molecules in ambient air. As a result, the strong bonding between adsorbed molecules and the $\mathrm{C}$ atoms on glassy graphene surface reasonably led to much weaker photoinduced desorption than that in the pristine counterpart. On the other hand, photogeneration of electron-hole pairs in glassy graphene with an intrinsic bandgap was more efficient compared with gapless graphene. Accordingly, photogeneration and recombination of electron-hole pairs dominated the photodetection process in glassy graphene, whereas the photoinduced sorption was the main contributor for photoresponse in pristine graphene. As a result, PPR was reasonably observed in glassy graphene photodetectors while NPR was obtained in the graphene devices. The persistent photoresponse observed in the graphene device can be attributed to the weak photogeneration of electron-hole pairs and strong role of molecular sorption mechanism. Moreover, in Fig. S4, the shift of charge neutral point toward negative voltage under $405 \mathrm{~nm}$ illumination and the bipolar behavior of 
photoconductivity observed in a graphene field-effecttransistor (FET) further verified the above photoresponse mechanisms. Detailed analysis can be found in the Supplementary information.

To measure the characteristics of the glassy graphene device operated under illumination at various wavelengths, the transient current dynamics under other visible and near-infrared laser illuminations are displayed in Fig. 3a, b, respectively. For all the wavelengths, the results demonstrate PPR with acceptable reproducibility of the photodetectors. The responsivities were calculated as $10.7,13,2.4$ and $2.2 \mathrm{~mA} \mathrm{~W}^{-1}$ for $450,520,635$ and $780 \mathrm{~nm}$ illumination, respectively. Accordingly, $D^{*}$ reaches $\sim 4.8 \times 10^{8}, 5.8 \times 10^{8}, 1.1 \times 10^{8}$ and $10^{8}$ Jones, respectively, comparable to the reported graphene photodetectors [51,52]. As for the photoresponse upon NIR 808 and $980 \mathrm{~nm}$ illumination, the photocurrent is more difficult to reach complete saturation as illustrated in Fig. 3b, which was also observed in previous photodetectors based on graphene oxide (GO) [52,53]. For the glassy graphene device, weak absorption in NIR range produces less photogenerated carriers. Given that the number of the traps retains at a constant value in the glassy graphene material, as a result, larger portion of photogenerated carriers could be trapped and longer response time is reasonably observed in the NIR range [53]. Additionally, the responsivity was calculated as 2.1 and $0.13 \mathrm{~mA} \mathrm{~W}^{-1}$ for 808 and $980 \mathrm{~nm}$ illumination, respectively.

Moreover, to examine the photoresponse of the glassy graphene device with larger thickness, power-dependent photoresponse characterization was carried out for the glassy graphene with the material thickness of $5.4 \mathrm{~nm}$ under 405-nm laser illumination, as illustrated in Fig. 3c. The decreasing trend of the peak intensity was also observed. As the incident power density increased, the photocurrent became larger accordingly, due to that more photoinduced carriers were produced and collected. The power-dependent photocurrent can be fitted by the power law $\left(I_{\mathrm{ph}} \propto P^{\beta}\right)$, where $\beta$ is the exponent [54]. $\beta$ was determined as 0.88 , as shown in Fig. $3 \mathrm{~d}$. The responsivity and specific detectivity under different power intensities were calculated and plotted in Fig. 3e, where $R$ and $D^{*}$ values decreased with increasing light intensity. The peak values of $R$ and $D^{*}$ are $48.8 \mathrm{~mA} \mathrm{~W}^{-1}$ and $\sim 1.5 \times 10^{8}$ Jones at a power density of $12.9 \mathrm{~mW} \mathrm{~cm}{ }^{-2}$, respectively. For further understanding of the photoresponse mechanisms, the schematic band structures of the glassy graphene
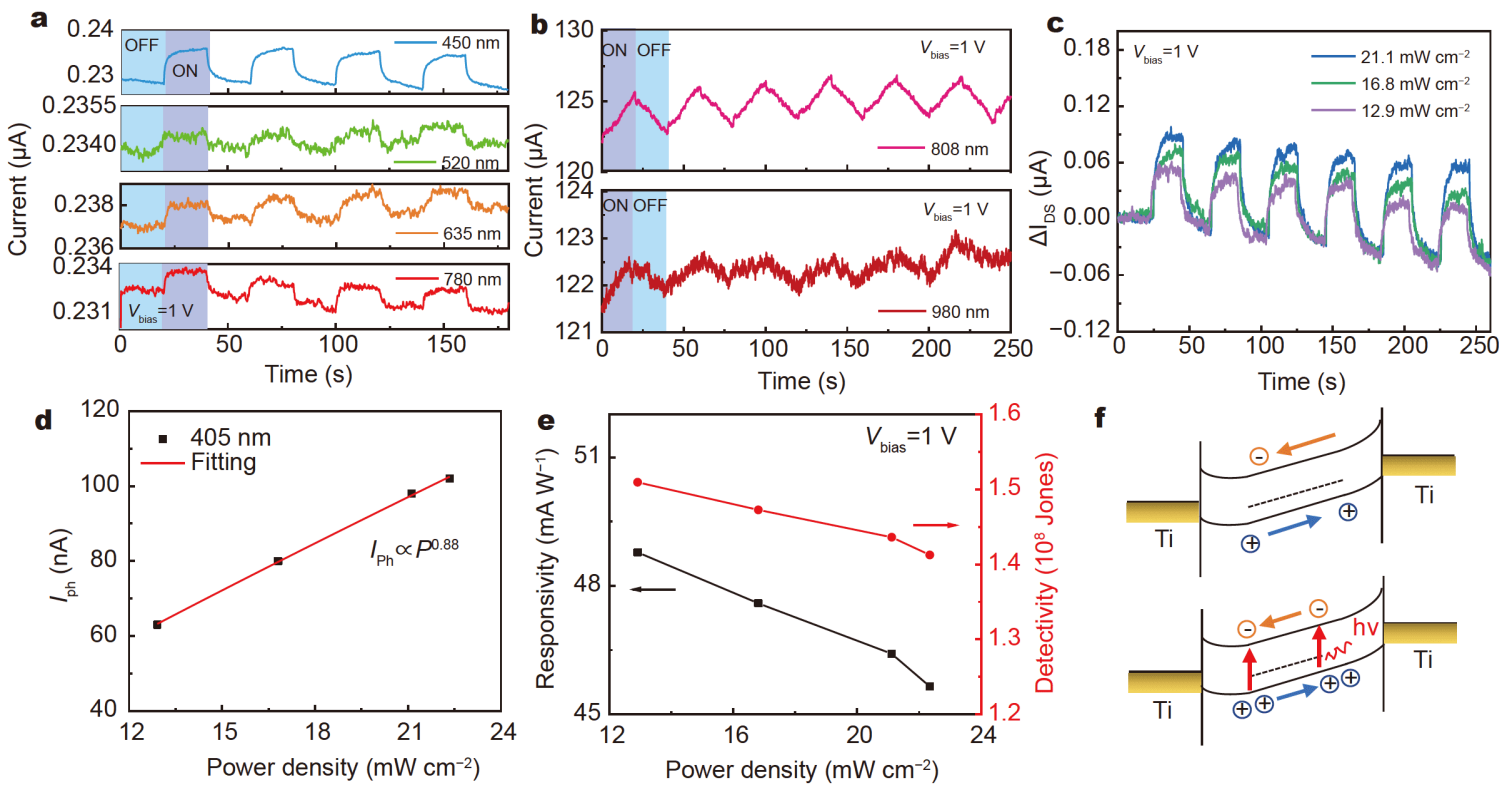

Figure 3 Photoresponse characteristics under additional vis-NIR illumination, power-dependent photoresponse performance and band structure of the glassy-graphene photodetector. (a) Periodic transient photoresponses of the glassy-graphene photodetector (1.5 nm) under 450, 520, 635, and $780 \mathrm{~nm}$ illumination, respectively. The applied constant voltage is $1 \mathrm{~V}$. (b) Photoresponse dynamics of another 1.5-nm glassy-graphene photodetector from the array illuminated by 808 - and $980-\mathrm{nm}$ laser. The applied constant voltage is $1 \mathrm{~V}$. (c) Power-dependent transient photoresponse of the glassygraphene photodetector $(5.4 \mathrm{~nm}$ ) with varied illumination power density at $405 \mathrm{~nm}$, under $1 \mathrm{~V}$ bias voltage. (d) The photocurrent versus power density, fitted by the power function. (e) The responsivity and specific detectivity as a function of power density. (f) Band structure of the glassygraphene photodetector under bias voltage in dark (top image), and under illumination (bottom image). 
photodetector are illustrated in Fig. 3f. It schematically shows the illumination OFF state (top image) and illumination ON state (bottom image) with a bias voltage applied on the photodetector. In Fig. S5, the work function of the $0.9-\mathrm{nm}$ glassy graphene was calculated as $\sim 3.2 \mathrm{eV}$ from the UPS spectrum. Due to the work function mismatch between $\mathrm{Ti}$ and glassy graphene, the energy band was bent at the interface. Under a bias voltage, some free carriers were collected by the two electrodes at dark. When excited, the photogenerated electron-hole pairs were separated by the built-in and external electric field, thus reducing the electron-hole recombination rates and increasing the charge carrier density dominantly [55]. Consequently, a larger number of charge carriers were collected by the two electrodes, displaying an increased photocurrent and PPR property.

\section{Electrical behavior via contacting VOC liquids}

Apart from the impressive photoresponsive performance, 2D glassy graphene also exhibits evident sensitivity and reproducibility for sensing VOCs in liquid. It is noted that the majority of liquid sensing studies reported the results at the time of contacting with liquid and ignored the subsequent variation in response, missing information related to subsequent diffusion and volatilization, especially for VOCs [56]. Nonetheless, the real-time electrical behavior recording the change during the whole process is significant for comprehensive sensing analysis and comparison.

VOC solvents, including acetone, IPA and methanol, were employed to contact with the glassy graphene devices for sensing. All the following measurements were conducted under ambient conditions, unless otherwise stated. The sensitivity was quantitatively determined by the current change ratio of $\left(I-I_{0}\right) / I_{0}(\%)$, in which $I_{0}$ is the initial channel current and $I$ is defined as the response current after the VOC liquid contacts the glassy graphene channels. Fig. 4a illustrates the dynamic current response characteristics of a $0.9-\mathrm{nm}$ FGD after contacting a $30-\mu \mathrm{L}$ acetone droplet at $V_{\text {bias }}=0.5 \mathrm{~V}$. For each full monitoring loop involved with droplet diffusion and volatilization at room temperature, sensing response to a VOC droplet could be divided into three stages. Once the acetone droplet contacted the glassy graphene channel (indicated by the red arrow in Fig. 4a), the FGD responded instantly with $52.7 \%$ increase in channel current, within $~ 80 \mathrm{~ms}$. The channel current was then plunged to a lower value and yet maintained a $28.6 \%$ increase in current. The initial stage, Stage I, lasted for about $5 \mathrm{~s}$. Afterwards, the channel current regained a slow and steady increase and saturated at 39.3\% (Stage II). Given the overall upward trend in the channel current, it was governed by acetone absorption in Stages I and II. The introduced lattice strain by SW defects has been reported to increase the bonding energy [57,58], which is confirmed by the binding energy for various gas chemicals resulting from SW defects larger than that from $\mathrm{sp}^{2}$-bonded carbons [50]. Accordingly, it is expected that the presence of SW defects in glassy graphene and the resulting intrinsic lattice strain could enhance the bonding between acetone molecules and glassy graphene, and thus improve acetone absorption. In the last stage, with the acetone droplet dispersing and volatilizing continuously in air, acetone desorption became dominant and the channel current started to decrease.

The response of the 0.9-nm FGD to acetone was further examined under a bias voltage varied from 0.01 to $1 \mathrm{~V}$ in Fig. $4 \mathrm{~b}$. When $V_{\text {bias }}=0.01 \mathrm{~V}$, the device exhibited a notable sharp initial response but large noise, as compared with $V_{\text {bias }}=0.5 \mathrm{~V}$ (Fig. 4a). As $V_{\text {bias }}$ increased, the corresponding saturated sensitivity value at Stage II (indicated by the red dashed lines) rose steadily from $16.1 \%$ to $67.7 \%$, while the initial response reduced sharply from $\sim 298.1 \%$ until it completely disappeared at $V_{\text {bias }}=1 \mathrm{~V}$. This observation validated that the acetone detection characteristics of the FGD were associated with bias voltage, which was able to effectively control the initial and saturated sensitivity. Additionally, a 2-nm FGD exposed to $30 \mu \mathrm{L}$ acetone solvent was studied, with the dynamic response shown in Fig. 4c. Similar to the 0.9-nm FGD, a sharp response was initially identified, followed by the steady stage and decay stage. Afterwards, the response current of the 2-nm FGD experienced irregular rise/fall processes, differing from that of the 0.9-nm FGD. This difference suggests the thickness of glassy graphene induces dissimilar "acetone-philicity" and might affect the acetone response characteristics. This assumption was further verified by a $25-\mathrm{nm}$ MGD as shown in Fig. $4 \mathrm{~d}$. Acetone detection of the 25-nm MGD showed rather different current characteristics, confirming thicknessdependent properties of the glassy graphene.

To clarify the specific acetone-philic characteristics on electrical response evolution, time-dependent sessile drop measurements of acetone were carried out on glassy graphene with different thicknesses, as shown in Fig. 4e. Initially, the contact angle on $0.9-\mathrm{nm}$ glassy graphene showed a sharp reduction from $\sim 25.4^{\circ}$ to $\sim 13.6^{\circ}$ within $\sim 69 \mathrm{~ms}$, after which the contact angles changed gradually with slight increments with time. It is noted that this time for the sudden change was very close to the $80 \mathrm{~ms}$ duration of the initial current spike of the FGD identified 

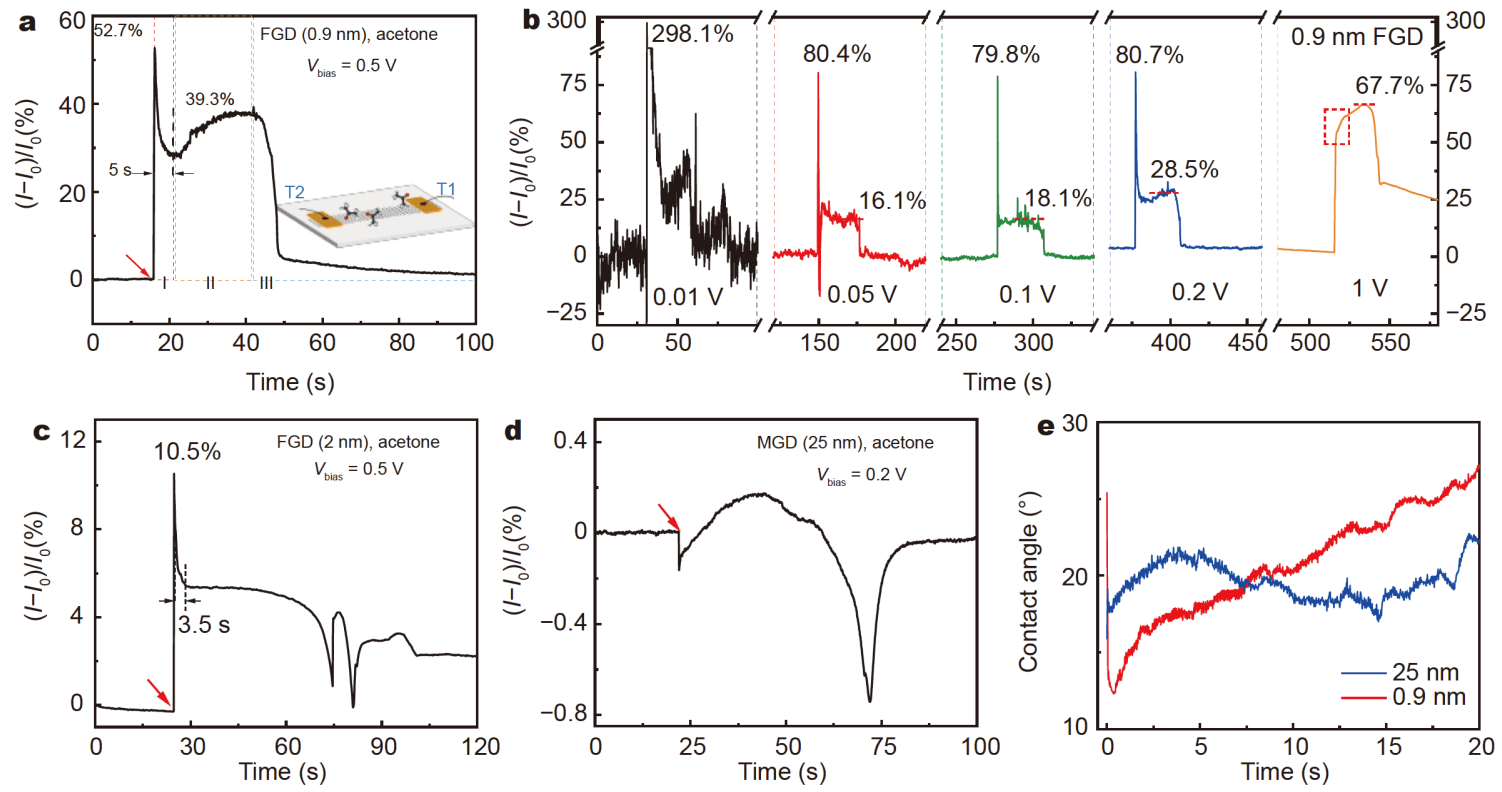

$\mathbf{f}$
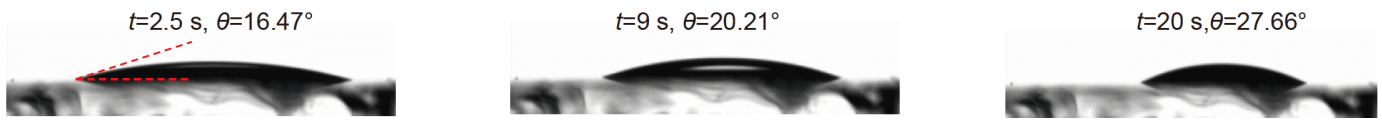

$\mathbf{g}$
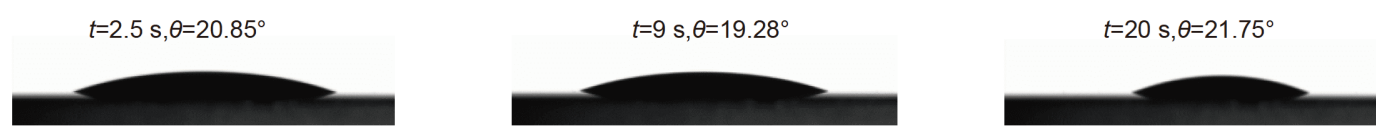

Figure 4 Liquid acetone sensing characteristics of the glassy-graphene devices and sessile drop measurement results for glassy-graphene/quartz. Dynamic detection response for 0.9-nm FGD at (a) $V_{\text {bias }}=0.5 \mathrm{~V}$ and (b) different $V_{\text {bias. }}$. Inset in (a) shows the schematic configuration of the glassygraphene device for sensing. Dynamic detection response for (c) 2-nm FGD and (d) 25-nm MGD. (e) Time-dependent contact angle variation of $30 \mu \mathrm{L}$ acetone on the surface of $0.9-\mathrm{nm}$ and 25-nm glassy-graphene/quartz, respectively. The selected time-lapse image series of acetone contact angles on (f) 0.9-nm and (g) 25-nm glassy-graphene on quartz. The red arrows in (a), (c), and (d) indicate the point of time when the channel was placed in contact with the acetone droplet.

in Fig. 4a. In contrast, the contact angle on the $25-\mathrm{nm}$ glassy graphene always showed a relatively stable fluctuation at $\sim 20^{\circ}$ since the acetone contacted the nanosheet surface. For both FGD and MGD, no sharp increase in response current was observed when the contact angles gradually changed. Therefore, it is reasonable to suspect that the sharp drop of current after the initial response of the FGD can be attributed to the abrupt contact angle change of the acetone droplet on the few-layer glassy graphene, which indicates higher acetone-philicity for the FGD at the very beginning. The abrupt change of acetone-philicity is very likely to destroy the uniform carrier distribution, leading to the non-even electric field and its subsequent redistribution. Thereby, the variation in response current can be effectively avoided through increasing the bias voltage which alters the electric field distribution, as validated in Fig. 4b. Additionally, the selected time-lapse images of an acetone droplet $(30 \mu \mathrm{L})$ on the $0.9-\mathrm{nm}$ glassy graphene/quartz are shown in Fig. 4f. It shows that the droplet contact angle became progressively larger. The droplet volume was obviously reduced at $t=20 \mathrm{~s}$, stemming from the continuous volatilization of acetone. Fig. 4g determines the case for 25$\mathrm{nm}$ glassy graphene on quartz, which reveals the relatively stable evolution of contact angles. Hence, the acetonephilic characteristics of glassy graphene and the resultant sensing response both exhibit thickness-dependent behavior.

To gain further insight into the detection mechanisms, the schematic diagrams of the energy band alignment for the glassy graphene devices were constructed and discussed. For FGD, the aligned band structure in thermal equilibrium is displayed in Fig. 5a (top), in which the barrier height $\phi 1$ and the space charge width $W 1$ are indicated. Acetone molecules were adsorbed via van der Waals force on the surface of glassy graphene and acted 
a
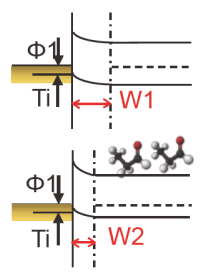

FGD

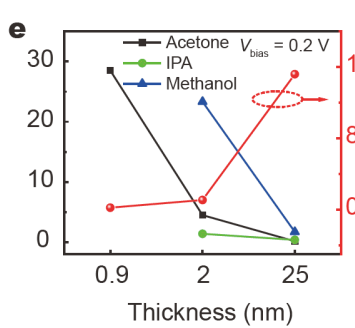

b
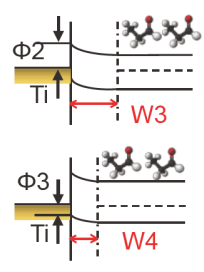

MGD

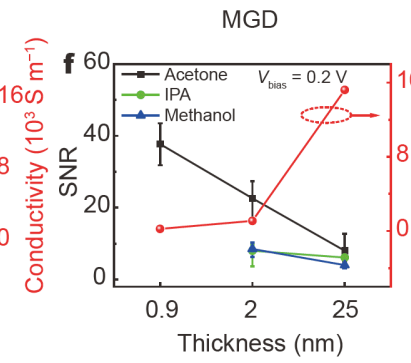

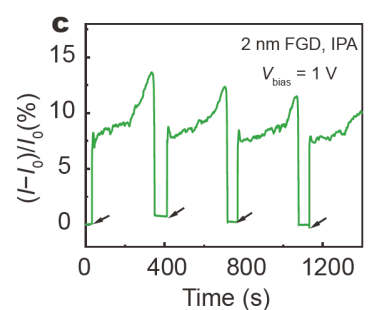
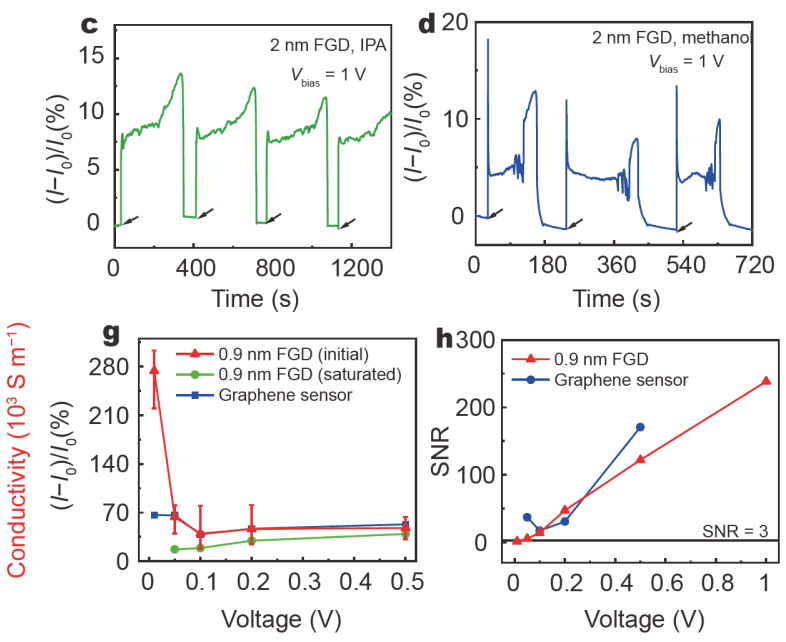

Figure 5 Band structures and sensing performance comparison results. Schematic diagrams of aligned band structures for (a) FGD and (b) MGD. The molecular models denote acetone molecules. The black, white and red balls represent carbon, hydrogen and oxygen atoms, respectively. Dynamic detection response of 2-nm FGD when sensing (c) IPA, and (d) methanol. The arrows denote the time when the glassy-graphene channel contacts the analyte. Thickness-dependent changing tendency of (e) current change ratio and (f) SNR when devices detected different VOC liquids, along with electrical conductivity. (g) Current change ratio comparison between FGD and graphene devices. (h) Voltage-dependent SNR comparison between FGD and graphene devices. The minimum required SNR of 3 is indicated.

as electron acceptors, similar to cases for other graphene's derivatives [48,59]. Accordingly, few-layer glassy graphene was p-doped after contacting with acetone, causing the Fermi level to further shift to the VBM. As shown in Fig. 5a (bottom), the space charge width $W 1$ was thereby reduced to $W 2(W 2<W 1)$, resulting in larger probability of tunneling and an enhanced channel current. This correlated well with the detected increase in channel current of FGD in Fig. $4 \mathrm{a}-\mathrm{c}$ during the acetone absorption processes. Furthermore, the channel current decreased during acetone desorption, resulting from the increased space charge width (tending to be $W 1$ again) caused by the gradually restored Fermi level of glassy graphene. According to former studies regarding multilayer graphene, doping from air-induced absorbate can only be effective in a few top layers due to the surface screening effect, and thus the other layers remain unaffected [60,61]. Accordingly, air exposure can presumably leave much less apparent impacts on multilayer glassy graphene than few-layer glassy graphene, resulting in negligible change of the Fermi level. Therefore, regarding the band structure of MGD, the scenario is different from that of FGD, as shown in Fig. 5b, with detailed sensing mechanism described in the Supplementary information.

Apart from acetone, glassy graphene devices were further examined by exposure to different VOC liquids, including IPA and methanol. Fig. 5c, d show the current response characteristics of the 2-nm FGD in contact directly with IPA and methanol, respectively, both of which differ from the current response when sensing acetone, suggesting good selectivity. The sensing mechanisms for IPA and methanol are similar to acetone, acting as electron acceptors as well, which is evident from the increased current upon exposure. The sharp initial response was observed during methanol detection, which could also be attributed to the initial abrupt change of contact angles. Additionally, IPA and methanol detection characteristics of the 25-nm MGD are illustrated in Fig. S6a, $\mathrm{b}$, respectively, and the noise could be decreased by applying large bias voltage. The repeatability and stability can be further improved by controlling the temperature or applying UV illumination to accelerate the VOC desorption process [62].

To further compare the impact of glassy graphene thickness on response properties, the corresponding sensitivity and SNR of FGD and MGD when monitoring VOC liquids were extracted and plotted as shown in Fig. $5 e, f$, together with the thickness-dependent electrical conductivity of the glassy graphene. SNR was calculated by Equation (1) below [63,64]:

$\mathrm{SNR}=\frac{A_{\max }}{\sigma_{\text {baseline }}}$.

Here, $\sigma_{\text {baseline }}$ is the standard deviation of baseline current change ratio before analyte delivery, and $A_{\max }$ is the saturated amplitude of current change ratio (sensitivity). 
An increase of the glassy graphene thickness from 0.9 to $25 \mathrm{~nm}$ significantly increases the conductivity, consistent with our previous study [32]. In contrast, both the sensitivity and SNR were reduced accordingly, which was observed for different VOC liquids. The mechanism can be mainly associated with the screening effect in multilayer glassy graphene. The increase in the thickness of glassy graphene would lead to an increase in conductivity, resulting in a larger initial channel current $I_{0}$. However, owing to the surface screening effect limiting the penetration of VOC molecules $[60,61,65]$, only a few uppermost layers of multilayer glassy graphene are involved in VOC detection, and thus it contributes to the response current close to the value obtained from the FGD. According to the definition of sensitivity, $\left(I-I_{0}\right) / I_{0}(\%)$, it is reasonable to obtain reduced sensitivity with increased thickness, and SNR is affected in a similar way. To sum up, few-layer glassy graphene is more sensitive for contacting with VOC liquids than thick glassy graphene.

Furthermore, the electrical behavior of a monolayer graphene device in the same configuration on $\mathrm{SiO}_{2} / \mathrm{Si}$ substrates was tested for comparison. The acetone detection characteristics of the graphene devices measured at $V_{\text {bias }}$ varied from 0.5 to $0.05 \mathrm{~V}$ are shown in Fig. S7, where negative sensing response was observed. The opposite sensing direction to glassy graphene is actually caused by different sensing mechanisms, with detailed analysis explained in the Supplementary information. In contrast to behaving as the acceptor for glassy graphene, acetone acted as the electron donor for the graphene channel, which was confirmed by the shift of transfer curves in a graphene FET. As shown in Fig. S8a, the Dirac voltage shifted from a large positive value $(>20 \mathrm{~V})$ towards the negative direction to $\sim 6.5 \mathrm{~V}$ upon the delivery of acetone, evidently indicating an $\mathrm{n}$-doping nature of acetone for graphene. Therefore, the carrier concentration of intrinsic p-type graphene decreased as more electrons transferred from acetone, resulting in a decreased sensing current. The similar electron donating property of acetone was also reported in previous studies $[66,67]$. Moreover, the acetone contact angle results are displayed in Fig. S8b, c. It is worth noting that neither rapid increase in current was observed during acetone monitoring processes under different bias voltages, nor there was an abrupt change of contact angles observed at the beginning of the time-dependent sessile drop measurement. This matched well with the case of glassy graphene devices, further implying the connection between the initial sharp increase in current and abrupt change of acetone contact angles.
Then, the sensing performance of the glassy graphene device $(0.9 \mathrm{~nm}$ FGD) was compared with that of a monolayer graphene device. Fig. $5 \mathrm{~g}$ shows the voltagedependent sensitivity of the 0.9-nm FGD, in comparison with the graphene device. For the FGD, both the initial response current and saturated current (indicated by the dashed lines in Fig. 4b) were extracted. Although the response of the FGD was comparable to the graphene one, the initial response of the FGD was noticeably larger than that of graphene at $V_{\text {bias }}=0.01 \mathrm{~V}$. Moreover, the sensitivity of the FGD was comparable or even superior to the reported liquid acetone sensors based on reduced GO $\left(\Delta R / R_{0}=\sim 45 \%\right.$ for bilayer $)$, graphene/cellulose nanocomposite $\left(\Delta R / R_{0}=2 \%-10 \%\right)$, and $\mathrm{Co}_{3} \mathrm{O}_{4}$-doped $\mathrm{ZnO}$ $\left(\Delta I / I_{0}=\sim 20 \%\right)[68-70] . R_{0}$ and $R$ represent the channel resistance before and after VOC liquids in contact with channels, respectively, and $\Delta R$ is the difference of $R$ and $R_{0}$. Additionally, in Fig. 5h, the voltage-dependent SNR of the $0.9-n m$ FGD exhibits a nearly linear variation tendency, compared with the graphene device. As $V_{\text {bias }}$ is enlarged to $0.05 \mathrm{~V}$, the SNR increases to $\sim 5.6$ and results in the valid sensitivity. Within the bias range of $0.1-0.5 \mathrm{~V}$, the SNR of the FGD is comparable to that of the graphene device and further increases up to $\sim 238.6$ at $V_{\text {bias }}=1 \mathrm{~V}$. Especially, the SNR reaches 48 at $0.2 \mathrm{~V}$ bias voltage, showing over $50 \%$ improvement to that of the graphene device. The obtained SNR values are tens of times larger than the critical value of 3 , competitive or even superior to other reported VOC sensors $[63,64,71]$. Therefore, the large-scale $0.9-\mathrm{nm}$ glassy graphene synthesized by the facile and cost-effective PAD method shares comparable sensitivity and reliability properties to graphene in liquid acetone detection. Good sensing performance of the FGD could be attributed to good conductivity, intrinsic bandgap and in-plane strain which resulted from SW defects in the glassy graphene [32,33]. An available bandgap could boost the sensitivity by balancing the electrical conductivity and change of conductivity after interacting with analyte [72]. In addition, defects are able to improve the chemical sensing ability for $2 \mathrm{D}$ materials by strengthening the interaction with analyte, and SW defects in the glassy graphene change the lattice constant, giving rise to in-plane strain, and thus enable more chemically active sites [57,58,73-75].

\section{CONCLUSIONS}

In summary, multifunctional devices based on large-scale 2D glassy graphene thin films were demonstrated for photodetection and chemical liquid sensing, both of which pose similar requirements on material properties, 
i.e., an appropriate bandgap and good electrical conductivity. With the distorted lattice-induced defects, glassy graphene was introduced with an appropriate bandgap, advantageous for photodetection and VOC liquid sensing. Due to the strong interaction between SW defects and electron-accepting molecules from air, photoinduced electron-hole generation and recombination dominated the photoresponse upon "on and off" illumination accordingly. PPR was achieved in glassy graphene devices, along with peak intensity declining due to the photoinduced molecule desorption, in contrast to the persistent NPR in the control graphene devices. Specifically, the glassy graphene device achieved a responsivity of $0.22 \mathrm{~A} \mathrm{~W}^{-1}$ and a specific detectivity of $\sim 10^{10}$ Jones under $405 \mathrm{~nm}$ illumination. The photoresponse performance upon additional vis-NIR illumination was also demonstrated for the photodetectors. In addition, for VOC liquid sensing, the continuous electrical behavior indicated VOC droplet diffusion and volatilization. Again thanks to the SW defects, strain and good conductivity, the glassy graphene device showed similar or even superior ability to pristine graphene device in acetone detection in terms of sensitivity and SNR. Specifically, the glassy graphene device showed $\sim 50 \%$ improvement of SNR over a graphene device at $0.2 \mathrm{~V}$ bias voltage. Moreover, the bias-voltage- and thickness-dependent acetone-philicity were examined, and later verified by the sessile drop measurements as well as IPA and methanol detection characteristics. The few-layer glassy graphene was verified more sensitive for sensing VOC liquids, while the performance of MGDs was limited by the screening effect. Importantly, the detailed detection mechanisms along with the schematic diagrams of the energy band alignment for the glassy graphene devices were analyzed and discussed. This study demonstrates the great potential of glassy graphene for high-performance multifunctional applications, particularly as one of the essential building blocks for integrated photodetection and VOC sensing.

Received 5 October 2020; accepted 31 December 2020; published online 9 March 2021

1 Hua Q, Sun J, Liu H, et al. Skin-inspired highly stretchable and conformable matrix networks for multifunctional sensing. Nat Commun, 2018, 9: 244

2 Gogurla N, Sinha AK, Santra S, et al. Multifunctional Au-ZnO plasmonic nanostructures for enhanced UV photodetector and room temperature NO sensing devices. Sci Rep, 2014, 4: 6483

3 Li J, Niu L, Zheng Z, et al. Photosensitive graphene transistors. Adv Mater, 2014, 26: 5239-5273
4 Liu CH, Chang YC, Norris TB, et al. Graphene photodetectors with ultra-broadband and high responsivity at room temperature. Nat Nanotech, 2014, 9: 273-278

5 Koppens FHL, Mueller T, Avouris P, et al. Photodetectors based on graphene, other two-dimensional materials and hybrid systems. Nat Nanotech, 2014, 9: 780-793

6 Ren A, Zou J, Lai $\mathrm{H}$, et al. Direct laser-patterned MXeneperovskite image sensor arrays for visible-near infrared photodetection. Mater Horiz, 2020, 7: 1901-1911

7 Bariya M, Nyein HYY, Javey A. Wearable sweat sensors. Nat Electron, 2018, 1: 160-171

8 Giraldo JP, Wu H, Newkirk GM, et al. Nanobiotechnology approaches for engineering smart plant sensors. Nat Nanotechnol, 2019, 14: 541-553

9 Fine GF, Cavanagh LM, Afonja A, et al. Metal oxide semiconductor gas sensors in environmental monitoring. Sensors, 2010, 10: $5469-5502$

10 Chuang CC, Prasannan A, Huang BR, et al. Simple synthesis of eco-friendly multifunctional silk-sericin capped zinc oxide nanorods and their potential for fabrication of hydrogen sensors and UV photodetectors. ACS Sustain Chem Eng, 2017, 5: 4002-4010

11 Shaikh SK, Ganbavle VV, Inamdar SI, et al. Multifunctional zinc oxide thin films for high-performance UV photodetectors and nitrogen dioxide gas sensors. RSC Adv, 2016, 6: 25641-25650

$12 \mathrm{Xu} \mathrm{H}$, Ren $\mathrm{A}, \mathrm{Wu}$, et al. Recent advances in 2D MXenes for photodetection. Adv Funct Mater, 2020, 30: 2000907

13 Chang H, Sun Z, Saito M, et al. Regulating infrared photoresponses in reduced graphene oxide phototransistors by defect and atomic structure control. ACS Nano, 2013, 7: 6310-6320

14 Makaram P, Owens D, Aceros J. Trends in nanomaterial-based non-invasive diabetes sensing technologies. Diagnostics, 2014, 4: $27-46$

15 Hakim M, Broza YY, Barash O, et al. Volatile organic compounds of lung cancer and possible biochemical pathways. Chem Rev, 2012, 112: 5949-5966

16 Broza YY, Haick H. Nanomaterial-based sensors for detection of disease by volatile organic compounds. Nanomedicine, 2013, 8 : 785-806

17 Bruen D, Delaney C, Florea L, et al. Glucose sensing for diabetes monitoring: Recent developments. Sensors, 2017, 17: 1866-1887

18 Yamada K, Ohishi K, Gilbert A, et al. Measurement of natural carbon isotopic composition of acetone in human urine. Anal Bioanal Chem, 2016, 408: 1597-1607

19 Geim AK, Novoselov KS. The rise of graphene. Nat Mater, 2007, 6: 183-191

20 Zhu J, Xu H, Zou G, et al. $\mathrm{MoS}_{2}-\mathrm{OH}$ bilayer-mediated growth of inch-sized monolayer $\mathrm{MoS}_{2}$ on arbitrary substrates. J Am Chem Soc, 2019, 141: 5392-5401

$21 \mathrm{Du} \mathrm{W}, \mathrm{Yu} \mathrm{P}, \mathrm{Zhu} J$, et al. An ultrathin $\mathrm{MoSe}_{2}$ photodetector with near-perfect absorption. Nanotechnology, 2020, 31: 225201

22 Lv Q, Yan F, Wei X, et al. High-performance, self-driven photodetector based on graphene sandwiched $\mathrm{GaSe} / \mathrm{WS}_{2}$ heterojunction. Adv Opt Mater, 2017, 6: 1700490

23 Wang X, Cui Y, Li T, et al. Recent advances in the functional 2D photonic and optoelectronic devices. Adv Opt Mater, 2019, 7: 1801274

24 Wei X, Yan F, Lv Q, et al. Fast gate-tunable photodetection in the graphene sandwiched $\mathrm{WSe}_{2} / \mathrm{GaSe}$ heterojunctions. Nanoscale, 2017, 9: 8388-8392

25 Mueller T, Xia F, Avouris P. Graphene photodetectors for high- 
speed optical communications. Nat Photon, 2010, 4: 297-301

26 Singh E, Meyyappan M, Nalwa HS. Flexible graphene-based wearable gas and chemical sensors. ACS Appl Mater Interfaces, 2017, 9: 34544-34586

27 Bae S, Kim H, Lee Y, et al. Roll-to-roll production of 30-inch graphene films for transparent electrodes. Nat Nanotech, 2010, 5: 574-578

28 Nair RR, Blake P, Grigorenko AN, et al. Fine structure constant defines visual transparency of graphene. Science, 2008, 320: 1308

29 Xia F, Mueller T, Golizadeh-Mojarad R, et al. Photocurrent imaging and efficient photon detection in a graphene transistor. Nano Lett, 2009, 9: 1039-1044

30 Park J, Ahn YH, Ruiz-Vargas C. Imaging of photocurrent generation and collection in single-layer graphene. Nano Lett, 2009, 9: 1742-1746

31 Schedin F, Geim AK, Morozov SV, et al. Detection of individual gas molecules adsorbed on graphene. Nat Mater, 2007, 6: 652-655

32 Dai X, Wu J, Qian Z, et al. Ultra-smooth glassy graphene thin films for flexible transparent circuits. Sci Adv, 2016, 2: e1601574

$33 \mathrm{Xu} \mathrm{H}$, Han X, Dai X, et al. High detectivity and transparent fewlayer $\mathrm{MoS}_{2}$ /glassy-graphene heterostructure photodetectors. Adv Mater, 2018, 30: 1706561

34 Gupta Chatterjee S, Chatterjee S, Ray AK, et al. Graphene-metal oxide nanohybrids for toxic gas sensor: A review. Sens Actuat BChem, 2015, 221: 1170-1181

35 Yuan W, Shi G. Graphene-based gas sensors. J Mater Chem A, 2013, 1: 10078-10091

36 Shen $\mathrm{K}, \mathrm{Li} \mathrm{X}, \mathrm{Xu} \mathrm{H}$, et al. Enhanced performance of $\mathrm{ZnO}$ nanoparticle decorated all-inorganic $\mathrm{CsPbBr}_{3}$ quantum dot photodetectors. J Mater Chem A, 2019, 7: 6134-6142

37 Di Bartolomeo A, Giubileo F, Iemmo L, et al. Transfer characteristics and contact resistance in $\mathrm{Ni}$ - and Ti-contacted graphene-based field-effect transistors. J Phys-Condens Matter, 2013, 25: 155303

38 Di Bartolomeo A, Santandrea S, Giubileo F, et al. Effect of backgate on contact resistance and on channel conductance in graphene-based field-effect transistors. Diamond Related Mater, 2013, 38: $19-23$

39 Anichini C, Czepa W, Pakulski D, et al. Chemical sensing with 2D materials. Chem Soc Rev, 2018, 47: 4860-4908

40 Xie C, Mak C, Tao X, et al. Photodetectors based on twodimensional layered materials beyond graphene. Adv Funct Mater, 2017, 27: 1603886

$41 \mathrm{Xu} \mathrm{H}$, Zhu J, Zou G, et al. Spatially bandgap-graded $\mathrm{MoS}_{2(1-x)} \mathrm{Se}_{2 x}$ homojunctions for self-powered visible-near-infrared phototransistors. Nano-Micro Lett, 2020, 12: 26

42 Lemme MC, Koppens FHL, Falk AL, et al. Gate-activated photoresponse in a graphene $\mathrm{p}-\mathrm{n}$ junction. Nano Lett, 2011, 11: 41344137

43 Kim CO, Kim S, Shin DH, et al. High photoresponsivity in an allgraphene $\mathrm{p}-\mathrm{n}$ vertical junction photodetector. Nat Commun, 2014, 5: 3249

44 Rathi S, Lee I, Lim D, et al. Tunable electrical and optical characteristics in monolayer graphene and few-layer $\mathrm{MoS}_{2}$ heterostructure devices. Nano Lett, 2015, 15: 5017-5024

45 Zhang W, Huang JK, Chen $\mathrm{CH}$, et al. High-gain phototransistors based on a CVD $\mathrm{MoS}_{2}$ monolayer. Adv Mater, 2013, 25: 3456-3461

46 Di Bartolomeo A, Giubileo F, Romeo F, et al. Graphene field effect transistors with niobium contacts and asymmetric transfer characteristics. Nanotechnology, 2015, 26: 475202
47 Piazza A, Giannazzo F, Buscarino G, et al. Graphene p-type doping and stability by thermal treatments in molecular oxygen controlled atmosphere. J Phys Chem C, 2015, 119: 22718-22723

48 Yang CM, Chen TC, Yang YC, et al. Enhanced acetone sensing properties of monolayer graphene at room temperature by electrode spacing effect and UV illumination. Sens Actuat B-Chem, 2017, 253: 77-84

49 Sun $\mathrm{P}, \mathrm{Zhu} \mathrm{M}$, Wang K, et al. Photoinduced molecular desorption from graphene films. Appl Phys Lett, 2012, 101: 053107

50 Qin X, Meng Q, Zhao W. Effects of Stone-Wales defect upon adsorption of formaldehyde on graphene sheet with or without $\mathrm{Al}$ dopant: A first principle study. Surf Sci, 2011, 605: 930-933

51 Safaei A, Chandra S, Shabbir MW, et al. Dirac plasmon-assisted asymmetric hot carrier generation for room-temperature infrared detection. Nat Commun, 2019, 10: 3498

52 Lai SK, Tang L, Hui YY, et al. A deep ultraviolet to near-infrared photoresponse from glucose-derived graphene oxide. J Mater Chem C, 2014, 2: 6971-6977

53 Qi X, Zou X, Huang Z, et al. Ultraviolet, visible, and near infrared photoresponse properties of solution processed graphene oxide. Appl Surf Sci, 2013, 266: 332-336

54 Shen K, Xu H, Li X, et al. Flexible and self-powered photodetector arrays based on all-inorganic $\mathrm{CsPbBr}_{3}$ quantum dots. Adv Mater, 2020, 32: 2000004

55 Zhang W, Chiu MH, Chen $\mathrm{CH}$, et al. Role of metal contacts in high-performance phototransistors based on $\mathrm{WSe}_{2}$ monolayers. ACS Nano, 2014, 8: 8653-8661

56 Yang $\mathrm{T}$, Zhang $\mathrm{H}$, Wang $\mathrm{Y}$, et al. Interconnected graphene/polymer micro-tube piping composites for liquid sensing. Nano Res, 2014, 7: 869-876

57 Dutta D, Wood BC, Bhide SY, et al. Enhanced gas adsorption on graphitic substrates via defects and local curvature: A density functional theory study. J Phys Chem C, 2014, 118: 7741-7750

58 An W, Wu X, Yang JL, et al. Adsorption and surface reactivity on single-walled boron nitride nanotubes containing Stone-Wales defects. J Phys Chem C, 2007, 111: 14105-14112

59 Masoud Darvish G, Hoda M, Azadeh K. Acetone adsorption on pristine and Pt-doped graphene: A first-principles vdW-DF study. Commun Theor Phys, 2015, 64: 576-582

60 Panchal V, Giusca CE, Lartsev A, et al. Atmospheric doping effects in epitaxial graphene: Correlation of local and global electrical studies. 2D Mater, 2016, 3: 015006

61 Miyazaki $\mathrm{H}$, Matsumoto R, Katagiri M, et al. $\mathrm{MoCl}_{5}$ intercalation doping and oxygen passivation of submicrometer-sized multilayer graphene. Jpn J Appl Phys, 2017, 56: 04CP02

62 Chen TC, Yang YC, Liu HL, et al. The effect of monolayer graphene on the UV assisted $\mathrm{NO}_{2}$ sensing and recovery at room temperature. Proceedings, 2017, 1: 461

63 Gao T, Woodka MD, Brunschwig BS, et al. Chemiresistors for array-based vapor sensing using composites of carbon black with low volatility organic molecules. Chem Mater, 2006, 18: 5193-5202

64 Tung TT, Castro M, Kim TY, et al. High stability silver nanoparticles-graphene/poly(ionic liquid)-based chemoresistive sensors for volatile organic compounds' detection. Anal Bioanal Chem, 2014, 406: 3995-4004

65 Pearce R, Iakimov T, Andersson M, et al. Epitaxially grown graphene based gas sensors for ultra sensitive $\mathrm{NO}_{2}$ detection. Sens Actuat B-Chem, 2011, 155: 451-455

66 Aliofkhazraei M, Ali N, Milne WI, et al. Graphene Science Handbook: Applications and Industrialization. Boca Raton: CRC 
Press, 2016

67 Zhang D, Liu A, Chang $\mathrm{H}$, et al. Room-temperature highperformance acetone gas sensor based on hydrothermal synthesized $\mathrm{SnO}_{2}$-reduced graphene oxide hybrid composite. RSC Adv, 2015, 5: 3016-3022

68 Jiang X, Yang T, Li C, et al. Rapid liquid recognition and quality inspection with graphene test papers. Glob Challenges, 2017, 1: 1700037

69 Gross MA, Sales MJA, Soler MAG, et al. Reduced graphene oxide multilayers for gas and liquid phases chemical sensing. RSC Adv, 2014, 4: 17917-17924

70 Rahman MM, Khan SB, Asiri AM, et al. Acetone sensor based on solvothermally prepared $\mathrm{ZnO}$ doped with $\mathrm{Co}_{3} \mathrm{O}_{4}$ nanorods. Microchim Acta, 2013, 180: 675-685

71 Li J, Lu Y, Ye Q, et al. Carbon nanotube sensors for gas and organic vapor detection. Nano Lett, 2003, 3: 929-933

72 Cui S, Pu H, Wells SA, et al. Ultrahigh sensitivity and layerdependent sensing performance of phosphorene-based gas sensors. Nat Commun, 2015, 6: 8632

73 Kou L, Du A, Chen C, et al. Strain engineering of selective chemical adsorption on monolayer $\mathrm{MoS}_{2}$. Nanoscale, 2014, 6: 51565161

74 Sahoo M, Wang J, Zhang Y, et al. Modulation of gas adsorption and magnetic properties of monolayer- $\mathrm{MoS}_{2}$ by antisite defect and strain. J Phys Chem C, 2016, 120: 14113-14121

75 Gautam M, Jayatissa AH. Detection of organic vapors by graphene films functionalized with metallic nanoparticles. J Appl Phys, 2012, 112: 114326

Acknowledgements This work was supported by the National Natural Science Foundation of China (61974014) and the EPSRC Future Compound Semiconductor Manufacturing Hub (EP/P006973/1). Dr. Hao Xu and Jian Guo thank the China Scholarship Council (CSC). We thank Sanjayan Sathasivam and Siyu Xiong for their help in the XPS, UPS and contact angle measurements, respectively.

Author contributions $\mathrm{Li} \mathrm{X}, \mathrm{Xu} \mathrm{H}$ and $\mathrm{Wu} \mathrm{J}$ conceived the idea and designed the experiments; Dai $\mathrm{X}$ and Zou G synthesized the glassy graphene thin films; Li X and Xu H performed the device fabrication; Li X, Shen K, Guo J, Parkin IP and Guo Z performed the material characterizations; Li X, Xu H and Li C performed the device measurements; Li X, Xu H, Choy KL, Liu H and Wu J analyzed the data; Li X and Xu H wrote the manuscript. All authors commented on the manuscript.

Conflict of interest The authors declare no conflict of interest.

Supplementary information Supporting data and experimental details are available in the online version of the paper.

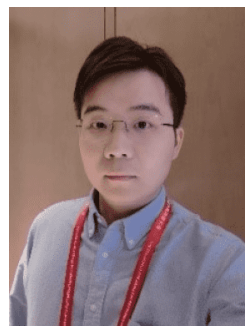

Hao Xu is a research Professor at the University of Electronic Science and Technology of China (UESTC). He received his Master's degree in microelectronics from Shanghai Institute of Microsystem and Information Technology, Chinese Academy of Sciences, and his $\mathrm{PhD}$ degree in photonics and nanotechnology from the University College London (UCL). His current research is focused on low-dimensional semiconductors and their applications for nanoelectronics and optoelectronics.

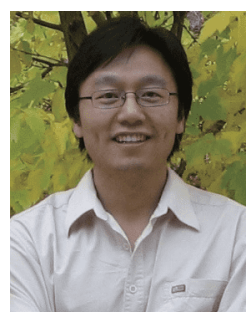

Guifu Zou received his $\mathrm{PhD}$ degree in chemistry from the University of Science and Technology of China in 2006. Following a postdoctoral position in Umea University (Sweden), he was appointed as a Director's Postdoctoral Fellow at Los Alamos National Laboratory in 2007. He joined Soochow University in 2011. His research focuses on inorganic thin films and emerging materials including 2D materials, perovskites, superconductors, and their potential applications in photovoltaics.

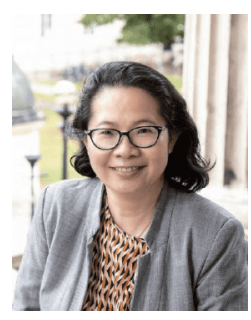

Kwang-Leong Choy is a Professor at UCL and Director of UCL Institute for Materials Discovery which was founded by her in 2014 . She obtained her $\mathrm{PhD}$ in materials science from the University of Oxford. She also held academic positions at the Imperial College London and the University of Nottingham. The current focus of Choy's group is nanomaterials, thin films and coatings for structural, functional and biomedical applications.

\section{多功能二维玻璃态石墨烯器件用于可见光-近红 外光探测与挥发性液体传感}

李潚 ${ }^{1,2 \dagger}$, 戴晓 ${ }^{3 \dagger}$, 徐浩 $^{1,2^{*}}$, 沈凯 ${ }^{1}$, 郭建 ${ }^{4}$, 李彩虹 ${ }^{2}$, 邹贵付 ${ }^{3 *}$, Kwang-Leong Choy ${ }^{5 *}$, Ivan P. Parkin ${ }^{4}$, 郭正晓 ${ }^{4,6,7}$, 刘会羿 ${ }^{1}$, 巫江 ${ }^{1,2}$

摘要 本文设计了基于二维玻璃态石墨烯的多功能器件. 与本征石 墨烯相比, 扭曲的晶格结构打开了玻璃态石墨烯的带隙, 表现出与 石墨烯类似甚至更优异的光电探测与化学传感性能. 由于玻璃态 石墨烯与空气中的小分子间较强的相互作用, 该器件受到光致脱 附的影响更小, 呈现出正的光响应. 在 $405 \mathrm{~nm}$ 的激光照射下, 器件 的响应率为 $0.22 \mathrm{~A} \mathrm{~W}^{-1}$, 探测率为 $\sim 10^{10}$ Jones. 此外, 玻璃态石墨烯 中的固有缺陷和应变可增强分析物的吸附, 获得良好的化学传感 性能. 玻璃态石墨烯器件探测丙酮的信噪比为 48 , 比石墨烯器件提 高了 $50 \%$ 以上. 此外, 对偏压和厚度有关的挥发性有机化合物 (VOC) 感测功能的分析表明, 少层玻璃态石墨烯更为敏感. 这项研 究表明玻璃态石墨烯在集成光电探测和化学传感多功能器件方面 具有巨大应用前景. 